\title{
Impulse Amplification in a Traveling-Wave Tube-II: Large Signal Physics
}

\author{
Mark C. Converse, Member, IEEE, and John H. Booske, Senior Member, IEEE
}

\begin{abstract}
The helix traveling-wave tube (TWT) is a vacuum electronic device which utilizes an electron beam to amplify electromagnetic waves. Although having a broad frequency gain bandwidth compared to other vacuum electron devices, the helix TWT has been used in relatively narrowband ways in its applications in communications and electronic countermeasures. Recent interest in ultrawideband (UWB) radio and radar, combined with the recent development of UWB helix TWTs, has stimulated interest in the amplification of very broadband multifrequency signals and impulses. A number of studies have been conducted on the harmonic and intermodulation effects of multiple signals in a TWT, but there has not been much investigation into impulse amplification. Utilizing a nonlinear time-domain model developed for this purpose, the response of a wideband (helix) TWT to an input Gaussian pulse is examined. Differences between the large signal response to positive and negative Gaussian inputs are examined, and it is shown that a positive impulse input yields better pulse shape preservation, higher saturation level, and possibly greater efficiency. The growth and saturation mechanisms of a positive input Gaussian are then investigated, showing distinct differences from steady-state growth and saturation mechanisms. This study indicates that the possibility of amplification of impulses in a TWT is very promising. The results and conclusions of this study generally apply to all types of TWTs with appreciable bandwidth (e.g., couple-cavity, folded waveguide). However, as the helix TWT has the largest bandwidth, it is the most interesting and is the specific TWT studied here.
\end{abstract}

Index Terms-Electron trapping, impulse amplification, impulse radar, impulse radio, microwave amplifier, time domain, traveling-wave tube (TWT), ultrawideband (UWB).

\section{INTRODUCTION}

$\mathbf{T}$ HE TRAVELING-WAVE tube (TWT) has been used as an electromagnetic amplifier since the 1940s, when the beneficial interaction between an electron beam and an electromagnetic wave guided by a helical waveguide was demonstrated [1]. During that time, it has seen much use as an amplifier in communications applications, radar, and electronic countermeasures. Until recently it was used in these applications as a narrowband amplifier, i.e., typically for single-frequency amplification. More recently it has been used to amplify multiple frequencies simultaneously, for which nonlinear effects are especially significant. A number of studies and models have been developed to examine these nonlinear effects (e.g., harmonics, sum frequencies, and intermodulation products) and have been rather successful. Now with recent

Manuscript received August 29, 2003; revised February 18, 2004.

The authors are with the Department of Electrical and Computer Engineering, University of Wisconsin, Madison, WI 53706 USA (e-mail: converse @ cae.wisc.edu; booske@engr.wisc.edu).

Digital Object Identifier 10.1109/TPS.2004.828788 increases in bandwidth, not only is multifrequency amplification of interest, but impulse amplification is also of interest. Some applications which might use pulse amplification include impulse radio [2], [3], impulse radar [4]-[6] and time-domain characterization of a TWT.

This paper describes an investigation of very large signal effects during impulse amplification in a wideband TWT. The study used only numerical simulation, since available experimental facilities were unable to access this regime. The numerical simulation employed a one-dimensional time-domain model previously described in [7].

In summary, the simulation study revealed the following important features of very large signal effects during impulse amplification in wideband TWTs.

- The amplitude evolution differs from conventional sinusoidal excitation.

- Saturation phenomena in general are different than sinusoidal excitation, including, for example, the absence of particle trapping.

- The very large signal response is qualitatively different for positive versus negative input pulse polarities.

Also, the explanations for these unique features of impulse amplification provide a clear and easily visualized confirmation of some basic principles governing the physics of conventional sinusoid amplification in a TWT, including the mechanism of bunching and the fact that once bunches form, they drive the new wave on the circuit, which then interacts with the beam to produce growth.

Before discussing the physics of large signal growth in a wideband TWT, it is helpful to review the growth and saturation of a single-frequency signal. For single-frequency operation within the gain bandwidth, the voltage versus position curve is initially constant for a short distance, while the electrons form into bunches and do not give net energy to the wave. After the electrons have began to bunch, the circuit voltage envelope experiences exponential growth, which would appear as a straight line on a log plot. Finally if the wave propagates for a long enough electrical distance, particle overtaking and trapping in the beam will occur, a maximum voltage will be reached, and saturation will occur. This is illustrated in Fig. 1. The maximum voltage at saturation is independent of the initial signal voltage. As the input voltage amplitude is increased, the axial location of saturation moves closer to the TWT input. This leads to a sort of interchangeability between input power and position as a drive curve "scan" variable. The voltage versus position trace for a larger input voltage is almost identical to that for a lower voltage, except that the onset of saturation is shifted closer to the TWT input. 


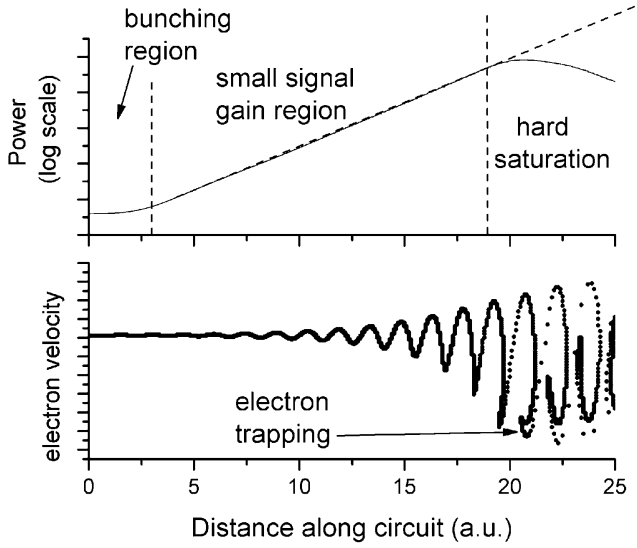

Fig. 1. Power and electron velocity along axis of TWT. The power graph indicates the bunching, exponential growth, and saturation regions. The electron velocity graph indicates saturation occurs when particle overtaking and trapping first begins to be seen.

\section{Difference Between Positive And Negative InPut PULSES IN THE LARGE SigNAL REGIME}

Simulations were used to study the response of a wideband TWT to both a positive and negative input impulse in both the small and large signal regime. The physical model used in the simulation is based on the eXperimental Wisconsin Northrop Grumman (XWING) TWT. This is a nominally $100-\mathrm{W}$, 2-6-GHz single-sever TWT (based on a production model) which was custom modified to allow greater diagnostic access to the fields and the beam for experimental research purposes [9]. Details of the physical model as well as information on the gain spectra can be found in [7]. There is one difference, however, in that for this study the sever was removed from the model. Fig. 2 shows the input signals whose magnitudes are scaled to give large and small input impulses.

As can be seen from Fig. 3, for the small signal case, a positive or a negative input leads to basically the same output signal, just flipped in polarity. In contrast, as seen in Fig. 4, for the large signal case, differences in output between the small and large signal case are much more substantial than just a change in polarity of the signal.

To investigate the reasons for this, the evolution of the wave voltage, electron velocities and beam density are compared for a positive and negative input Gaussian impulse. Figs. 5 and 6 show the two cases. The input voltage peak for this particular example is $11.4 \mathrm{~V}$.

From the figures, it can be seen that initially the voltages, particle velocities, and density are similar but flipped in polarity for both positive and negative input cases. However, as the disturbance propagates along the axis, differences can be seen to develop.

In Fig. 5, the negative voltage input impulse develops a positive voltage peak on the leading edge, seen in Fig. 5(a). By Fig. 5(b), two more positive voltage peaks develop on the front and back of the pulse, and a second negative voltage peak develops for a total of five peaks (three positive, two negative). Between Fig. 5(c) and 5(d), the three positive voltage peaks appear to saturate, while the two negative peaks continue to grow. Meanwhile, between Fig. 5(a) and 5(b), the electrons decelerate at the negative slope portions of the voltage waveform and accelerate at the positive slope portions. By Fig. 5(c), the decelerated electrons begin to fall back and charge overtaking is observed. Finally, in Fig. 5(a) and 5(b), the electron density develops two bunches (negative peaks) with a rarefaction or antibunch (positive peak) in between. The bunches grow and become tighter until they resolve into two sharp spikes by Fig. 5(c).

In Fig. 6, the initially positive voltage impulse develops a negative voltage peak in Fig. 6(a) and a second positive peak in Fig. 6(b). In Fig. 6(c)-6(d), all three peaks grow, but the negative peak grows more quickly than the positive peaks. Electrons are decelerated at the negative slope regions of the voltage pulse and accelerated at the positive slope regions. By Fig. 6(c), the decelerated electrons fall back, and charge overtaking occurs. Finally, in Fig. 6(a) and 6(b), the density is characterized by the formation of a single bunch in between two antibunches. The bunch is quite intense and localized by Fig. 6(c). In fact, in Fig. 6(d), one can see that the beam density has been completely rarefied in front of the main density bunch. Virtually all charge has been pulled back from this portion of the beam into the main bunch or pushed forward into a smaller, secondary bunch in Fig. 6(d).

The most obvious differences between the two cases are observed in the circuit voltage and beam density. Specifically, the initially negative voltage pulse ends up with two large negative voltage peaks (and three smaller positive peaks) and two dominant density bunches. In contrast, the initially positive voltage pulse ends up with one large negative voltage peak (and two smaller positive voltage peaks) and one dominant density bunch. In addition to the differences in the number of voltage peaks and density bunches, in both cases the outcome favors predominantly negative polarity voltage disturbances, regardless of the initial voltage impulse polarity.

The reason for this favoring of negative voltage disturbances may be found in the makeup of the beam. Since the beam consists of only negatively charged particles, one can only get negative charge density bunches. What we call antibunches are not positive density bunches but only indicate regions of the absence of negative charge. This means that while negative bunches can theoretically continue to grow indefinitely, an antibunch is limited in magnitude by the dc current density. Once all the electrons are gone from a region, the antibunch can no longer grow. Now, as negative voltage disturbances are associated with negative density bunches, positive voltage disturbances are associated with antibunches. This means that there will be a limit to the extent to which a positive voltage disturbance can grow, and we see this in the saturation of the positive voltage peaks in the evolution of both the positive and negative input impulse signals.

There are probably multiple reasons for the different responses to input voltage polarity. However, the primary reason appears to originate in the electric fields for the original input voltage pulse.

In the case of the negative input voltage pulse (Fig. 5), the electric field at the front of the pulse accelerates electrons while the electric field on the backside decelerates electrons. This produces two initial electron density bunches, one in front of the pulse and one behind it. The beam current is dominated by the 

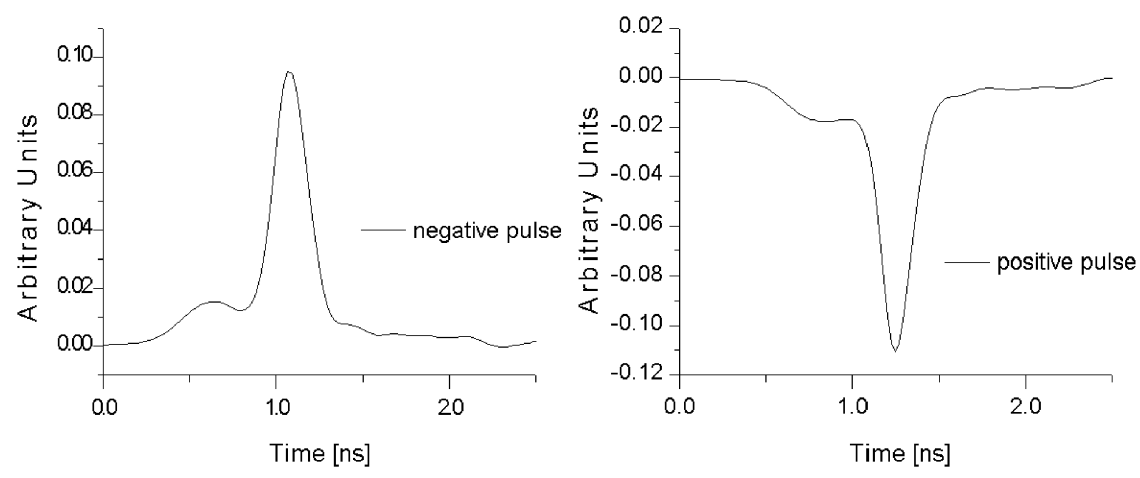

Fig. 2. Voltage versus time of positive and negative input signals used in the models.
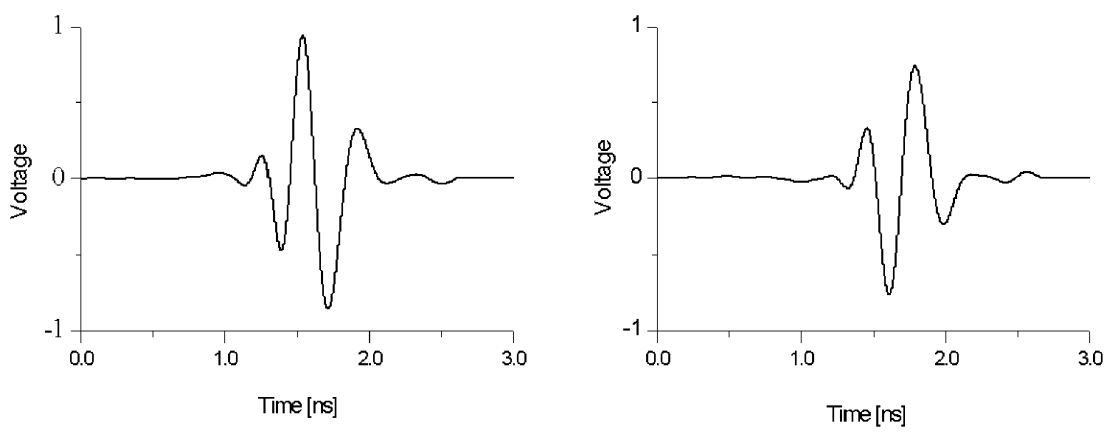

Fig. 3. Voltage versus time for small signal positive (left) and negative (right) input pulse.
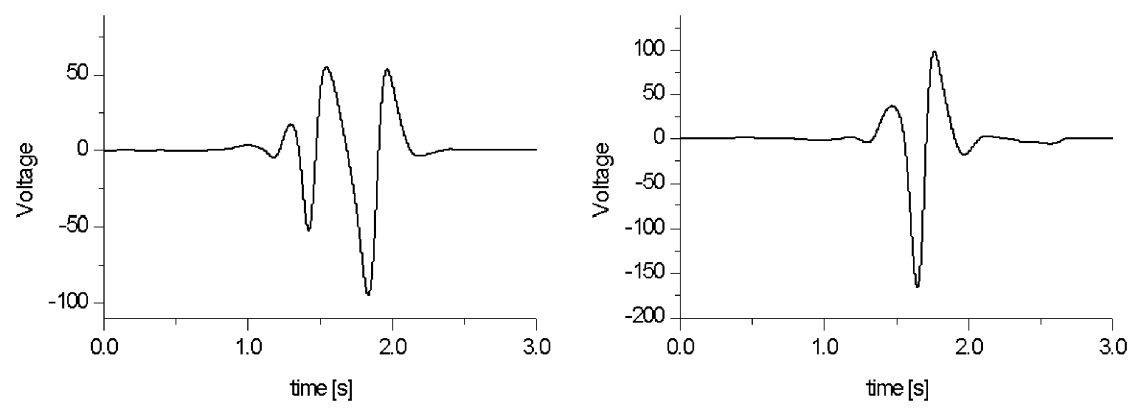

Fig. 4. Voltage versus time for large signal positive and negative input pulse.
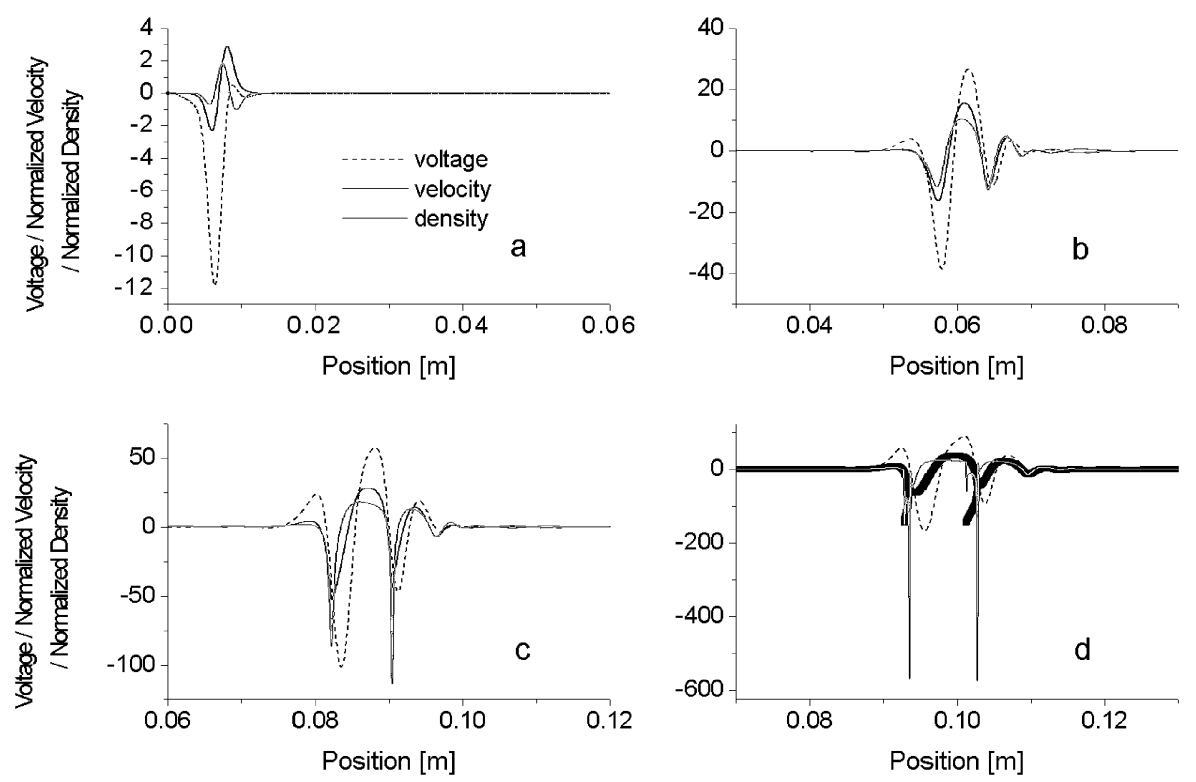

Fig. 5. Voltage (black dashed), scaled particle velocity (black, thin and thick), and scaled density (gray) versus $z$ at various points along the axis of the TWT for a negative Gaussian input pulse. Scaled velocity $=(v-u 0) / 1 \times 10^{5}$. Scaled density $=\left(\right.$ density $\left.-\rho_{0}\right) * 2000$. 

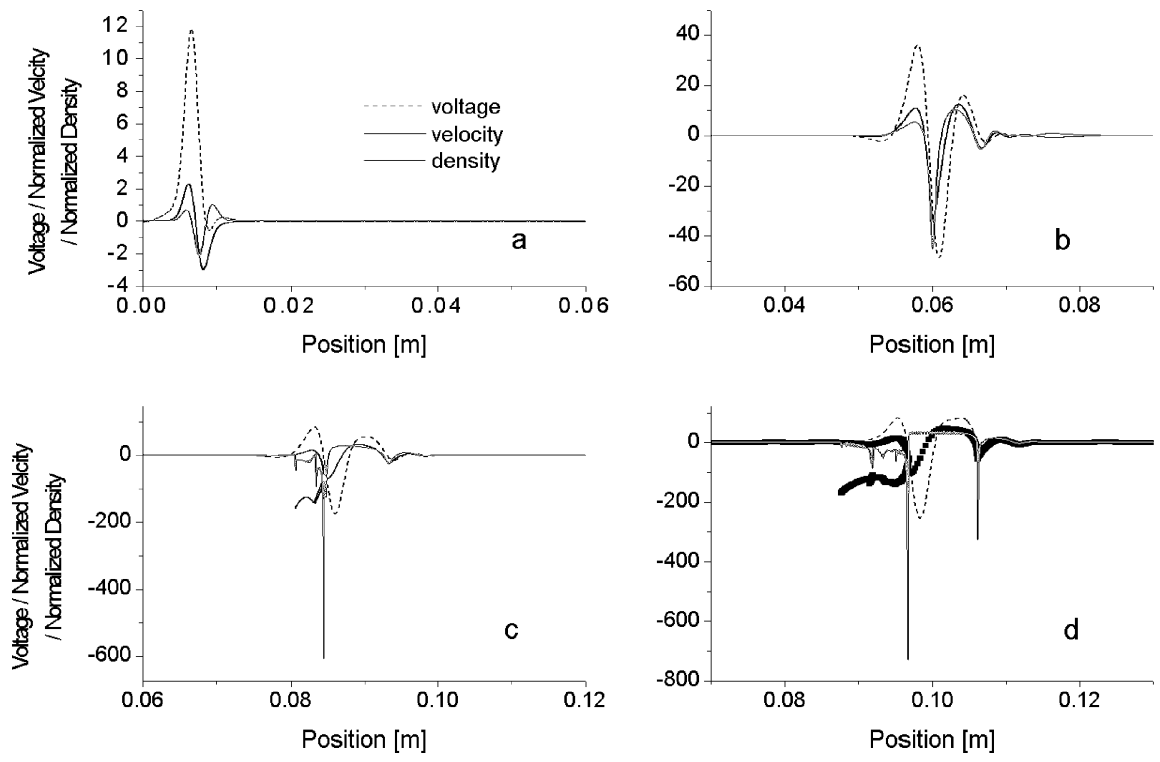

Fig. 6. Voltage (black dashed), scaled particle velocity (black, thin and thick), and scaled density (gray) versus $z$ at various points along the axis of the TWT for a positive Gaussian input pulse. Scaled velocity $=(v-u 0) / 1 \times 10^{5}$. Scaled density $=\left(\right.$ density $\left.-\rho_{0}\right) * 2000$.

electron density and so, the beam current acquires two bunches, one in front and one behind the original pulse. Just as in conventional harmonically excited TWT theory, the modulated beam current induces a matching modulated image current on the helix which results in the emergence of two negative voltage peaks. The density bunches end up concentrating in the decelerating field portion of the induced negative voltage peaks, and the two bunches and the two negative voltage peaks grow together as the electrons in each bunch are further decelerated.

In the case of the positive input voltage pulse (Fig. 6), the electric field at the front of the pulse is decelerating, while the electric field on the back of the pulse accelerates electrons. This results in a single bunch, localized near the front edge of the input pulse. Again, the modulated density produces an equivalently modulated beam current that induces an equivalently modulated image current in the helix, which causes a negative polarity circuit voltage peak to form in front of the original positive peak. The single electron density bunch interacts with the decelerating electric field on the backside of this negative voltage peak, and the bunch and the new negative voltage peak grow together.

Thus, a conclusion is that the positive polarity input impulse appears to result in a narrower, broader band (negative) output voltage pulse than the initially negative polarity input pulse.

It also appears that there are opportunities for extremely efficient pulsed energy extraction and anomalously high voltage pulse gain. Compare, for example, the velocity phase space results at the back of each disturbance-i.e., for the main bunch in Fig. 6, or the trailing larger bunch in Fig. 5-with the velocities for the smaller bunch at the front of the disturbance in Fig. 5. As electrons are decelerated at the back of the disturbance, they will not encounter a voltage peak or electric field that could reaccelerate them and return some energy back to the electron beam. These trailing bunch electrons simply trail farther and farther behind the disturbance, remaining at a lower energy. This suggests that an anomalously greater efficiency could be obtained for an amplification of a single positive pulse than for a single harmonic or multifrequency steady-state signal. In the latter cases, as electrons in each bunch decelerate and fall back, they encounter the voltage signal propagating behind them which can reaccelerate them and return energy back to the electron beam. This classic electron trapping mechanism for signal saturation with sinusoidal excitation is clearly evident in Fig. 1.

It is also speculated that the same isolated interaction with a single voltage peak enables unusually complete accumulation of electrons in a bunch and results in the unusually complete density rarefaction or "hole burning" in the TWT electron beam [see, for example, $z \sim 10 \mathrm{~cm}$ in Fig. 6(d)].

\section{Saturation Mechanisms for a Positive GaUssian INPUT PULSE}

One mechanism for saturation in single-frequency steadystate operation involves particle trapping. As the electrons in the bunch give up energy to the wave, their velocity decreases to a level below the hot phase velocity of the wave, and they fall out of the decelerating region and into the accelerating region of the wave. Due to the periodic nature of the wave, there is always an accelerating region behind a decelerating region. When the electrons fall into the accelerating region, they gain energy and velocity (taking energy from the wave) and move out of the accelerating region back into the decelerating region, where they lose energy and velocity again, and the cycle continues.

Consider the positive Gaussian input pulse. This is a nonperiodic signal; there are no preceding or trailing pulses. One would, therefore, expect that there might be differences in saturation mechanisms compared to steady-state sinusoidal excitations. However, before proceeding with an investigation of saturation, a metric for characterizing the pulse gain needs to be chosen. There does not seem to be one universal metric to describe the response of the TWT to a pulse (or transient) input. For single-frequency excitation, metrics used to describe the response of the TWT are small signal gain versus frequency, saturated power, and power-in-versus-power-out drive curves. 


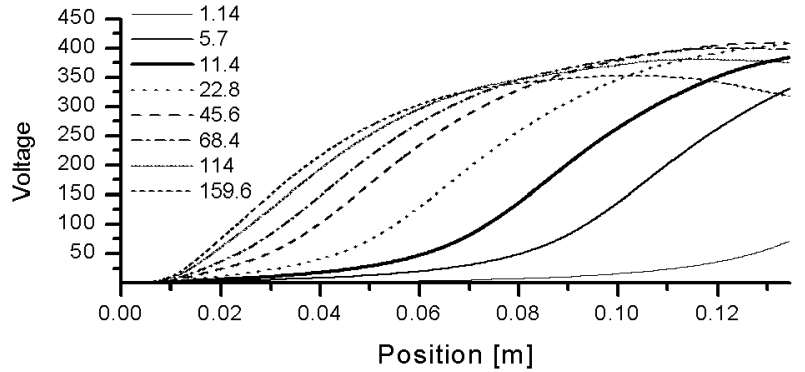

Fig. 7. Plot of absolute value of negative voltage peaks versus position for a various positive Gaussian input voltage levels. Legend gives value of positive voltage peak input.

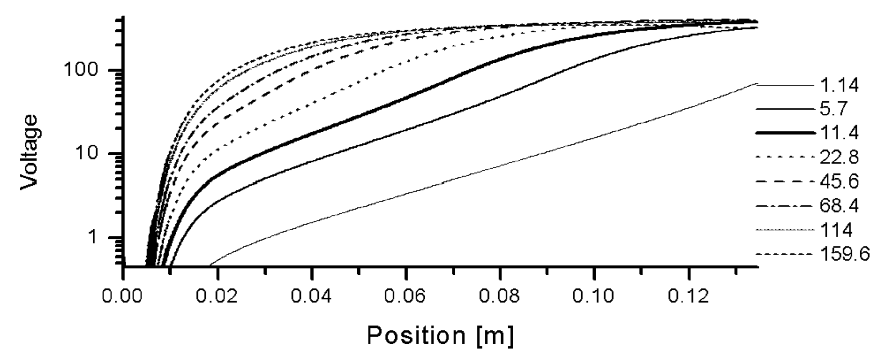

Fig. 8. Log plot of absolute value of negative voltage peaks versus position for a various positive Gaussian input voltage levels. Legend gives value of positive voltage peak input.

For a number of reasons [8], these metrics do not work when attempting to describe the response of the TWT to impulses. There are other metrics which could be considered-for example, total energy in versus total energy out or instantaneous peak power in versus instantaneous peak power out. These metrics, however, will not work for all cases, and it appears as though a metric needs to be chosen on a case-by-case basis. For this case, we will chose peak instantaneous absolute voltage. There is a single input peak and a distinct output peak, so peak instantaneous absolute voltage should provide a reasonably unambiguous measure of voltage pulse gain.

Fig. 7 shows the peak negative voltage versus position along the axis for a number of positive input voltage impulse amplitudes. Fig. 8 shows the same plot in logarithmic scale.

From Figs. 7 and 8, there are several immediately apparent differences between these voltage-versus- $z$ curves and similar curves that one would obtain for single-frequency operation.

Recall that for single-frequency operation, the maximum voltage at saturation is independent of the initial signal voltage. As the input voltage amplitude is increased, the axial location of saturation moves closer to the TWT input. This leads to a sort of interchangeability between input power and position as a drive curve "scan" variable. The voltage-versus-position trace for a larger input voltage is almost identical to that for a lower voltage, except that the onset of saturation is shifted closer to the TWT input.

This conventional drive curve shape and interchangeability of input power and $z$ is not evident for the impulse-excited case. From Fig. 8 it is evident from the two distinct slopes in any particular trace that the shape of the peak envelope is different from that for the single-frequency operation. Unlike the single-frequency excitation case with a single straight slope (in a logarithmic scale) which transitions into saturation, for the impulse-excitation case there are two straight slopes- the first one very steep, the second shallower, which then transitions into saturation. Also, as the input voltage changes, the shape for higher input voltages is different from that of lower input voltages. A trace of higher input voltage cannot simply be shifted in $z$ to overlay a lower input voltage trace. Finally, looking at Fig. 7, it can be seen that changing the input impulse voltage results in a different maximum voltage at saturation. The lower input voltages yield maximum voltage peaks of over $400 \mathrm{~V}$, while the higher voltage inputs yield only $350-375 \mathrm{~V}$ at saturation. Here we have defined the saturation point as the point at which the voltage envelope reaches a maximum value and the saturation regime, as the regime in which the growth of the wave is no longer exponential but begins to taper off.

A closer examination of the envelope structures in Figs. 7 and 8 shows a region of negligible or very small signal in all cases until about $0.5 \mathrm{~cm}$ along the axis. This is because the envelope signal shows the absolute value of the negative pulse, and for the positive input Guassian impulse, the negative peak does not begin to show significant development until about $0.5 \mathrm{~cm}$. This distance is approximately the same for all input impulse voltages, and, perhaps not coincidentally, this represents the distance for the input impulse to completely enter the simulation.

The next region on the trace is the first straight slope. In this region, the density bunches and antibunches start small and grow quickly. It can be seen in Fig. 9 that the density bunches grow much faster than the voltage or velocity modulation [Fig. 9(a)-9(c)]. By Fig. 9(d), however, the density no longer grows significantly faster than the voltage or velocity modulation, and for Fig. 9(e) and 9(f), it grows at about the same rate as the voltage and velocity. This change in the growth rate of the density is consistent with the transition region from large growth of the voltage envelope to smaller growth of the voltage envelope at about $1 \mathrm{~cm}$ along the TWT, for the 11.4-V input impulse case. From this it seems likely that initially the density bunches are small enough that the space-charge fields do not affect the electron velocities significantly. Eventually the bunches grow intense enough that their self-fields start to retard further electron accumulation, and a transition into a new, slower growth rate is seen. This could be analogous to the bunching region seen in the single-frequency steady-state excitation case.

After the transition region between steep and shallow slopes in the trace, the voltage grows exponentially until a distance of approximately $7-7.5 \mathrm{~cm}$ along the axis for the $11.4-\mathrm{V}$ input impulse case. At this point, the effect of the space-charge electric field and the circuit voltage combine to slow the decelerated electrons down to a velocity slower than that of the wave disturbance. Consider Fig. 10. In Fig. 10(a)-10(c), we see the density bunch growing, while the electron velocities in the region of the density bunches slow down. At Fig. 10(d), the signs of the development of the trailing electrons can be seen. In Fig. 10(e) and 10(f), there are definite signs of trailing electrons and these trailing electrons are starting to affect the density profile. Up until Fig. 10(c) and 10(d), the electrons, even after deceleration near the negative voltage peak, retained enough velocity to pass through the wave disturbance and continue beyond the voltage peaks. The cold-phase velocity of the wave in the gain 

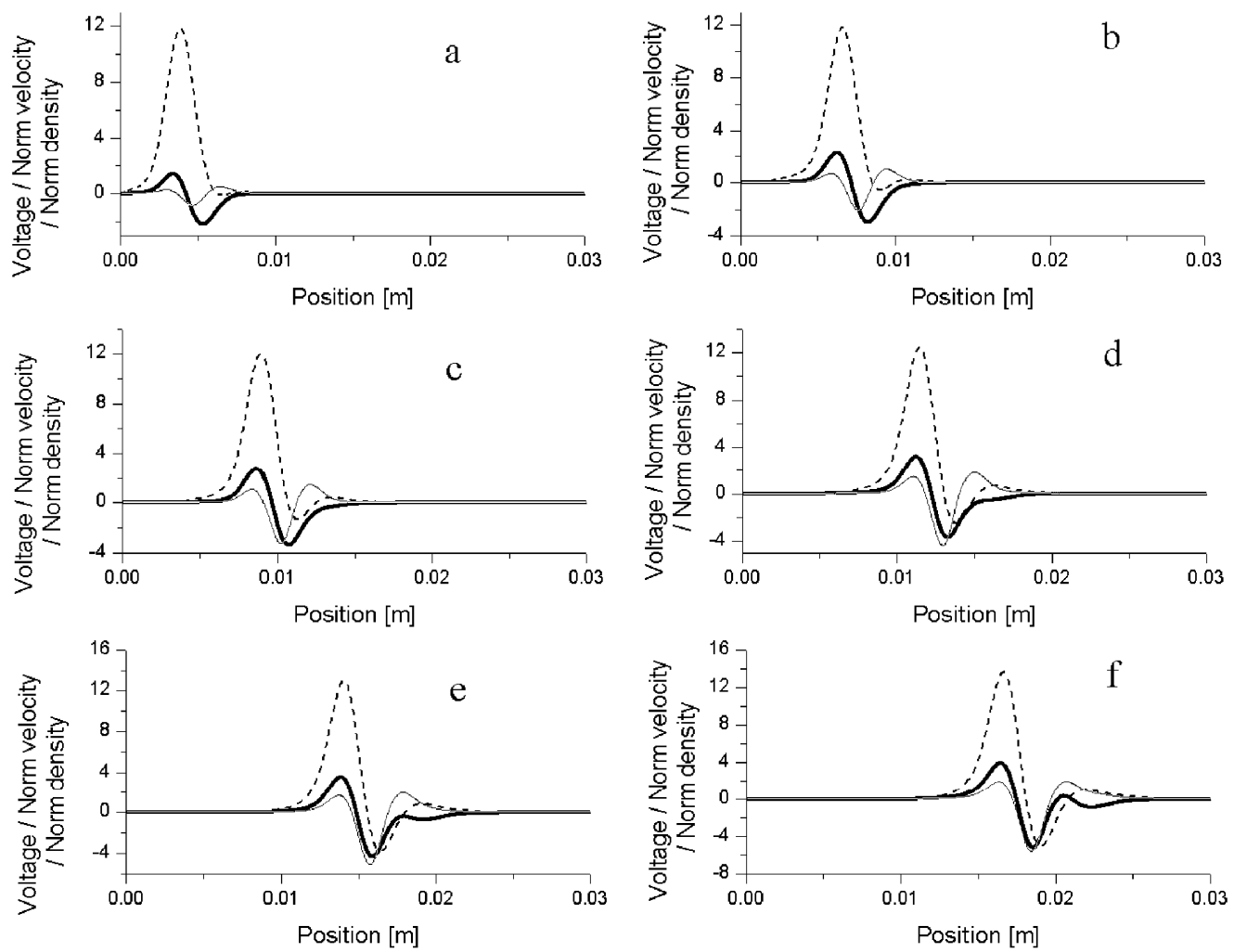

Fig. 9. Voltage (black dashed), scaled particle velocity (black thick), and scaled density (gray) versus $z$ at various points along the axis of the TWT for a positive Gaussian input pulse with input peak equal to 11.4 . Scaled velocity $=(v-u 0) / 1 \times 10^{5}$. Scaled density $=\left(\right.$ density $\left.-\rho_{0}\right) * 2000$.
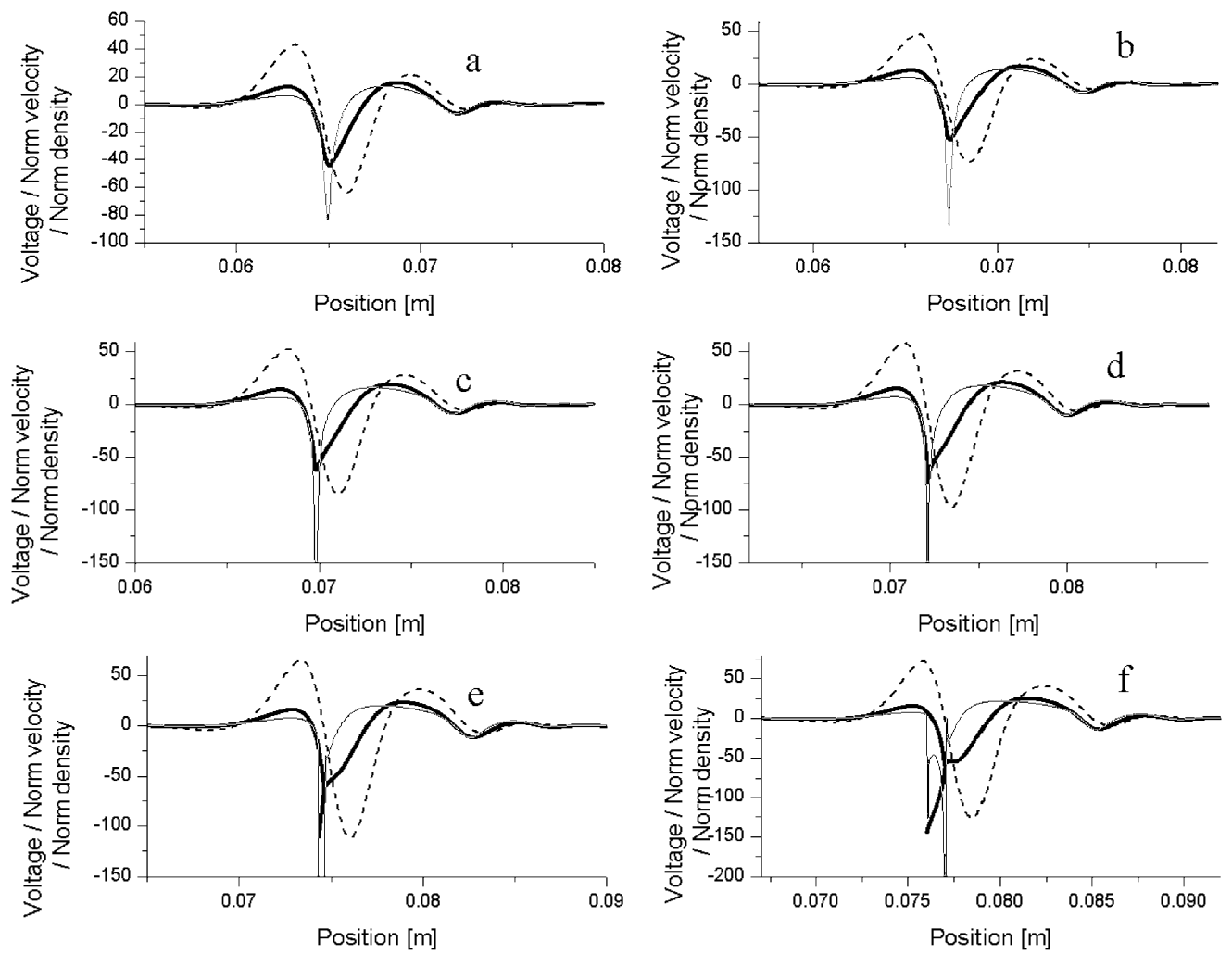

Fig. 10. Voltage (black dashed), scaled particle velocity (black thick), and scaled density (gray) versus $z$ at various points along the axis of the TWT for a positive Gaussian input pulse with input peak equal to $11.4 \mathrm{~V}$. Scaled velocity $=(v-u 0) / 1 \times 10^{5}$. Scaled density $=\left(\right.$ density $\left.-\rho_{0}\right) * 2000$.

regime varies from approximately $2.47 \times 10^{7}-2.54 \times 10^{7} \mathrm{~m} / \mathrm{s}, \quad 2.5 \times 10^{7} \mathrm{~m} / \mathrm{s}$. Therefore, somewhere in this area we would exand the electron velocity at a normalized value of -55 is about pect the electrons to begin to be unable to pass through the nega- 

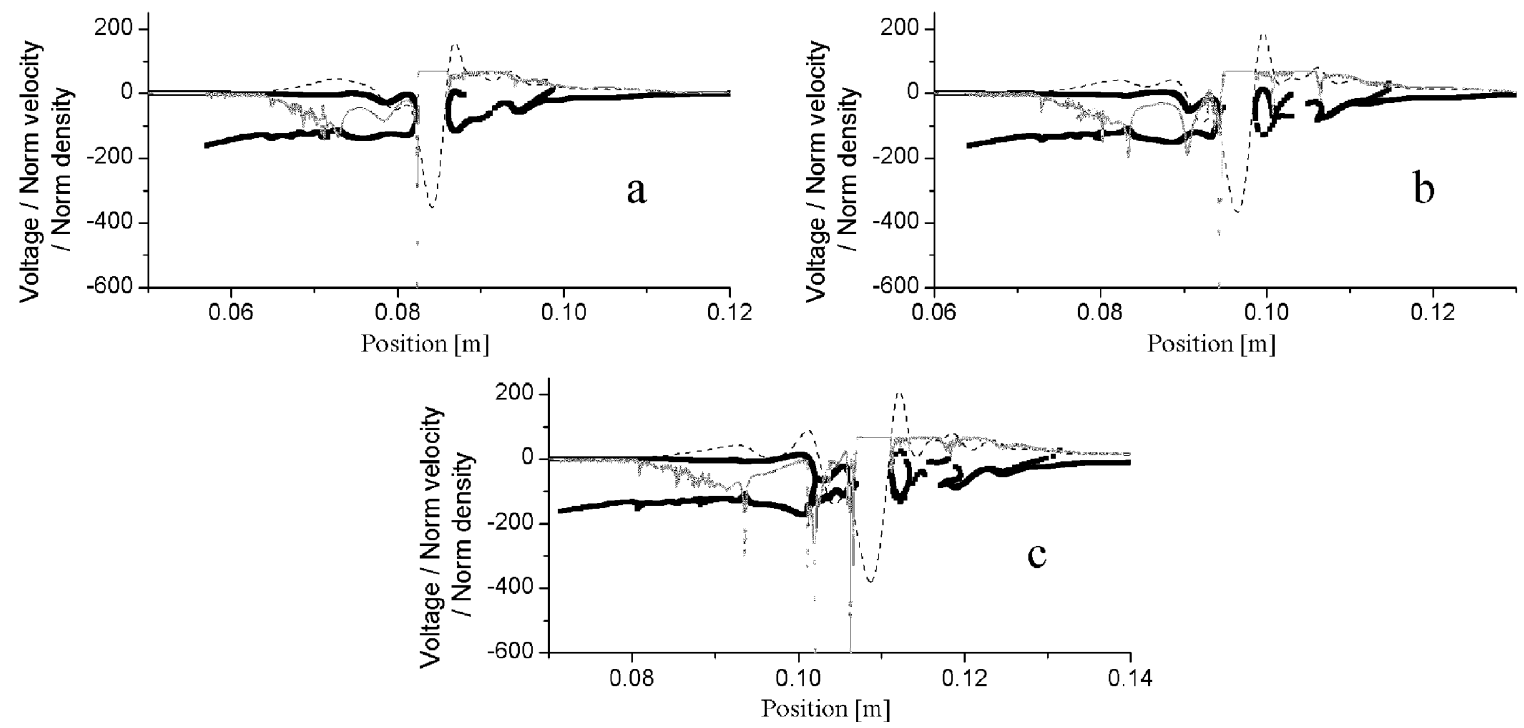

Fig. 11. Voltage (black dashed), scaled particle velocity (black thick), and scaled density (gray) versus $z$ at various points along the axis of the TWT for a positive Gaussian input pulse with input peak equal to $114 \mathrm{~V}$. Scaled velocity $=(v-u 0) / 1 \times 10^{5}$. Scaled density $=\left(\right.$ density $\left.-\rho_{0}\right) * 2000$.

tive voltage peak region and begin to collect in the density bunch or even begin to fall behind. This point in $z$, at which some of the electrons slow to a velocity below the wave disturbance velocity, is approximately the region in which the slope of the voltage envelope begins to change and become shallower. This is the beginning of the saturation regime. The voltage peak continues to grow in this regime, albeit at a considerably slower rate.

As seen in Fig. 7, for input pulse voltages less than or equal to $11.4 \mathrm{~V}$, the signal does not even reach the saturation point. So for subsequent discussion of the saturation effects, the 114-V input impulse case will be considered (Fig. 11). Consider the voltage, beam velocity, and beam density as the signal approaches its saturated value at approximately $11 \mathrm{~cm}$ (as seen in Fig. 7). It can be seen from Fig. 11(a) that at $8.5 \mathrm{~cm}$, there is a significant trail of electrons behind the negative pulse. Auxiliary voltage peaks are beginning to form behind the main negative peak, and there are also significant voltage disturbances in front of the main negative peak. Near the first voltage peak in front of the dominant negative peak, particle trapping can begin to be seen. At $9.5 \mathrm{~cm}$ [Fig. 11(b)], there is definite particle trapping near the positive voltage disturbance region proceeding the main negative peak. Other peaks behind the main negative peak have become distinct and are beginning to show signs of particle trapping as well. When the main peak has reached $11 \mathrm{~cm}$ [Fig. 11(c)], there is particle trapping both in front of and behind it, and the circuit voltage shows a number of oscillations both before and after the negative pulse. It is interesting to note that most of these leading and trailing edge voltage oscillations are associated with a positive dc voltage bias component.

It appears as though in some sense saturation is similar to saturation for the case of single-frequency excitation, in that particle trapping plays a significant role, although perhaps not in exactly the same way. Voltage pulse saturation appears to involve the trapping of particles in the auxiliary voltage peaks following the dominant negative peak. This particle trapping behind the main pulse appears to occur at the expense of the growth of the main negative voltage peak.

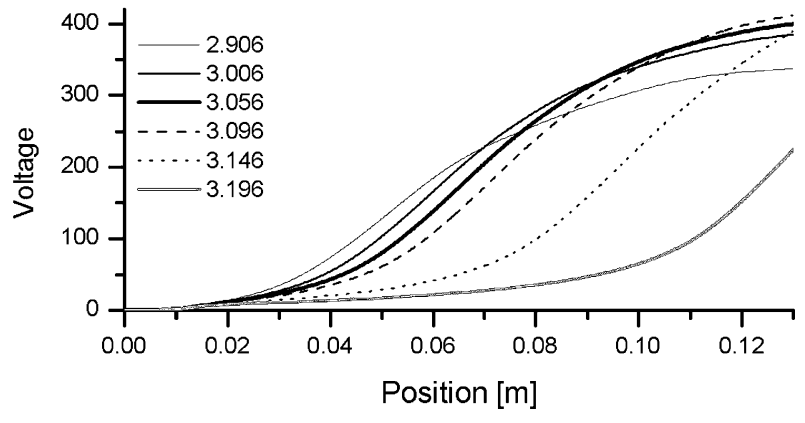

Fig. 12. Plot of absolute value of negative voltage peaks versus position for the 22.8-V input impulse Gaussian case for a number of initial electron velocities. Legend gives the value of the initial electron velocity $/ 1 \times 10^{7}$.

\section{SATURATion Mechanism ARgument Strengthened By CONSIDERING VELOCITY DETUNING}

The effect of varying the beam velocity for the $22.8-\mathrm{V}$ input impulse has been investigated. Fig. 12 shows the voltage envelope for a number of initial beam velocities. Note that $3.056 \times 10^{7} \mathrm{~m} / \mathrm{s}$ is the beam velocity used in all of the other studies described in this paper. From the figure, it can be seen that lower electron velocities result in voltage peaks that initially grow more quickly but saturate sooner and at lower levels than the cases with faster initial electron velocities. This seems consistent with the explanation of saturation. Recall the transition to the saturation regime begins near the point where the electron velocity, in the region of the negative pulse, decreases to a velocity slower than the wave disturbance velocity. This leads to trailing electrons, which fall behind the disturbance and result in a lower growth rate.

For lower initial beam velocities, the difference between the initial beam velocity and the wave disturbance velocity is smaller than for higher beam velocities. Therefore, the deceleration required to bring the electron velocity down below the wave disturbance velocity is less. This means the electric fields due to the negative voltage pulse and the space-charge electric 
fields do not need to be as large to achieve this deceleration, and so the cases with lower initial electron velocities would be expected to saturate nearer the input of the TWT.

\section{SUMMARY}

This study has yielded a basic understanding of the physics behind the growth of a Gaussian pulse in a TWT. Differences between positive input Gaussian impulses and negative Gaussian impulses have been ascribed to beam density bunches and the tendency for negative charge bunches to grow. Also, an explanation of the different regimes and the transitions between regimes is given for the growth curve of a negative voltage peak for a positive Gaussian impulse input. The mechanisms that result in saturation of this signal are also discussed. It is found that the growth and saturation mechanisms show distinct differences from harmonic excitation. This basic understanding has led to extrapolations of the benefits of impulse operation. Some of the currently perceived benefits include increased efficiency due to the lack of reacceleration of trailing electrons at saturation, the maintenance of the impulse shape when a positive input Gaussian impulse is used, and a greater maximum output voltage than that found for steady-state operation.

\section{REFERENCES}

[1] J. R. Pierce, Traveling Wave Tubes, ser. Bell Laboratory Series D. Princeton, NJ: Van Nostrand, 1950.

[2] M. Z. Win and R. A. Scholtz, "Impulse radio: How it works," IEEE Commun. Lett., vol. 2, pp. 36-38, Feb. 1998

[3] — , "Ultra-wide bandwidth time-hopping spread-spectrum impulse radio for wireless multiple-access communications," IEEE Trans. Commun., vol. 48, pp. 679-691, Apr. 2000.

[4] M. G. M. Hussain, "Ultra-wideband impulse radar-An overview of the principles," IEEE Aerosp. Electron. Syst. Mag., vol. 13, pp. 9-14, Sept. 1998.

[5] D. L. Black, "An overview of impulse radar phenomenon," IEEE Aerosp. Electron. Syst. Mag., vol. 7, pp. 6-11, Dec. 1992.

[6] D. Daniels, "Applications of impulse radar technology," in Proc. Radar 97, pp. 667-672.

[7] M. Converse, J. H. Booske, and S. C. Hagness, "Impulse amplification in a helix traveling-wave tube-I: Simulation and experimental validation," Trans. Plasma. Sci., vol. 32, pp. 1040-1048, June 2004.

[8] M. Converse, "Investigation of the mechanisms of pulse amplification in helical traveling wave tubes," Ph.D. dissertation, Univ. Wisconsin, Madison, 2003.
[9] M. Wirth, "Experimental investigations of a custom-made, helical traveling wave tube amplifier," M.S. thesis, Univ. Wisconsin, Madison, 2002

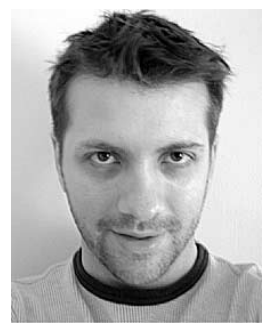

Mark C. Converse (SM'91-M'96) received the B.S. degreee in electrical engineering and the M.S. and $\mathrm{Ph} . \mathrm{D}$. degrees from the University of Wisconsin, Madison, in 1996, 1999, and 2003, respectively.

During his graduate studies, he was engaged in plasma processing research involving damage evaluation/analysis and mitigation during the etching process. After obtaining his M.S. degree, he began research in microwave vacuum electronics investigating the impulse response of the helix traveling-wave tube. He is currently a Postdoctoral Scholar, University of Wisconsin, working on projects involving the microwave detection and treatment of breast and liver cancer using microwaves. His research interests include electromagnetic interactions with materials, electrical/biological interfaces, and organic electronics.

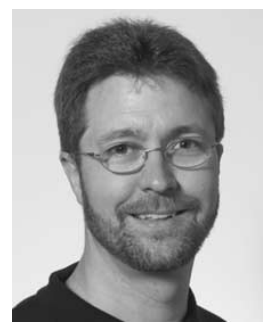

John H. Booske (S'82-M'85-SM'93) received the $\mathrm{Ph} . \mathrm{D}$. degree in nuclear engineering from the University of Michigan, Ann Arbor, in 1985.

From 1985 to 1989 , he was a Research Scientist at the University of Maryland, College Park. In 1990, he joined the faculty of the University of Wisconsin, Madison, where he is a Professor of Electrical and Computer Engineering. In 2001, he was appointed Director of the University of Wisconsin's Materials Science Program. His research interests include the experimental and theoretical study of coherent electromagnetic radiation sources and their applications, with emphasis in the RF, microwave, millimeter-wave, and terahertz regimes. Recent research activities include vacuum electronics, microwave and RF heating of materials, biological/biomedical applications of microwave and RF fields, high current density field emission cathodes, and ultrashallow junction fabrication in silicon CMOS transistors.

Prof. Booske received the University of Wisconsin Vilas Associate Award for research excellence and the U.S. National Science Foundation Presidential Young Investigator Award. He has been honored with many teaching awards, including the University of Wisconsin Chancellor's Distinguished Teaching Award and the Benjamin Smith Reynolds Award for Excellence in Teaching Engineering. He served as Codirector of a U.S. Department of Defense MURI99 consortium on innovative microwave vacuum electronics and as Director of a MURI04 consortium on cathode field emission and RF window breakdown in high-power microwave sources. 